\title{
NUMERICAL MODELLING OF THE DYNAMICS OF THE GALACTIC HALOS IN THE COLLIDING GALAXIES
}

\author{
S.S. Khrapov ${ }^{1}$, A.V. Khoperskov ${ }^{1}$, V.I. Korchagin ${ }^{2}$ \\ ${ }^{1}$ Volgograd State University, Volgograd, Russian Federation \\ ${ }^{2}$ Southern Federal University, Rostov-on-Don, Russian Federation \\ E-mails: khrapov@volsu.ru,khoperskov@volsu.ru,vkorchagin@sfedu.ru
}

\begin{abstract}
Based on parallel three-dimensional simulation of N-body and gas self-consistent dynamics, we study the behavior of hot coronal gas in the colliding galaxies with "live" dark matter halos. We model a few scenarios of the galactic collisions including "bull-eye" and non-central ones, and use different values of the initial velocities of the colliding galaxies. Taking into account the self-gravity, we demonstrate that the collision of gaseous and stellar components does not lead to the formation of a gaseous "protogalaxy" observed in some numerical simulations. Also, we show that about sixty percent of hot halo gas is expelled into intergalactic space during the collision. Numerical simulations show that a considerable amount of gas (up to $70 \%$ for a bull-eye collisions) exchanges between two colliding galaxies.

Keywords: Multi-GPU; OpenMP-CUDA; GPU-Direct; NVIDIA TESLA; N-body; SPH; colliding galaxies.
\end{abstract}

\section{Introduction}

Norman and Silk [1] suggested that a substantial amount of hot enriched gas, produced during early stages of galactic evolution, holding within massive extended halos can be released into the intracluster medium during the collisions. A presence of hot gas in the massive elliptical galaxies as well as in the halos of the spiral ones is confirmed by observations (see e.g. [2]). Observations of massive elliptical galaxies, for example, demonstrate that their X-ray luminosity is non-stellar in nature [3] confirming its hot gaseous origin. The existence of a hot extended Milky Way halo has been inferred from observations of OVI absorption lines in high-velocity clouds.

The baryon budget of the Universe shows significant evolution from $z \sim 3$, and results in an apparent baryon deficit today [4,5]. At high redsfhifts most of the baryonic mass is observed in the Ly $\alpha$ forest $[4,5]$, while at low redshifts roughly 60 percent of the baryons may exist in form warm-hot $\left(10^{6}-10^{7} \mathrm{~K}\right)$ intergalactic medium. Gas is driven into the IGM by galaxy mergers. A merger rate of galaxies evolves rapidly, decreasing from $\sim 50$ percent at $z \sim 3$ to $\sim 5-10$ percent at $z \sim 1$. The collisions of galaxies in the galactic clusters is therefore the important link in understanding the budget of baryons in the Universe. Yet there was not paid much attention to the enrichment of intergalactic medium by the galactic collisions.

Singha and Holley-Bockelmann [6] simulated the dynamics of hot halo gas during a merger of galaxies with the initial velocities of merging galaxies comparable or smaller to the intrinsic velocity dispersion of the individual galaxies. A typical velocity dispersion of galaxies within a cluster is of order of a few hundred kilometers per second, so a highspeed collision between the galaxies within a cluster is much more probable. It is relevant therefore to model the dynamics of hot halo gas during the collisions with more realistic 
values of the initial velocities of collisions comparable to the velocity dispersion of galaxies within a cluster.

The dynamics of hot gaseous halo in the colliding galaxies was addressed in papers $[7,8]$. These authors considered a few scenarios of the collisions assuming that gravitational potentials of galaxies. Besides fixed gravitational potentials of the galaxies, $[7,8]$ assumed that galaxies move towards each other with constant velocities unchanged during the collision, and assume also that masses of the collisionless halos and the gaseous ones are equal. Under these assumptions the authors find that certain intitial conditions can lead to the formation of a gaseous "protogalaxy".

Hwang and Park [9] using N-body + SPH simulations studied the distant encounters between an early and late type galaxies aiming to clarify the dynamics of hot halo gas. Hwang and Park [9] find that during the encounter with closest separation about $100 \mathrm{kpc}$ between the two galaxies, about $5 \%$ of cold gas is transferred from one galaxy to another.

As it was mentioned above, galaxies in clusters experienced a number of close interactions in the past. To understand the budget of barions in the Universe, it is important therefore to study the consequences of close galactic collisions. In this paper we model numerically the dynamics of colliding galaxies that have live dark matter particles as well as hot halo gas, and vary the initial velocities and the impact parameters aiming to determine the amount of halo gas expelled to the intergalactic medium during the collisions. We aim also to explore the possibility of the formation of gaseous "proptogalaxy" during the galactic collision in more realistic self-consistent model when each colliding galaxy is modeled by "live" collisionless and gaseous components joined by common gravity.

Another important difference from the numerical simulations performed by $[7,8]$ is that we calculate N-body interactions between the particles directly avoiding thus a possible influence of errors associated with approximate calculation of gravitational force in TreeCode.

\section{Equations and Equilibrium Model}

To simulate the dynamics of colliding galaxies, we use a set of equations that self-consistently describe the behavior of a collisionless-gaseous halos of galaxies. A collisionless component (dark matter and stars) of galaxies has mass $M_{c}$ and consists of $N_{c}$ particles, and gaseous component with mass $M_{g}$ has $N_{g}$ particles. The set of equations for a collisionless and the gaseous components of galaxies consisting of $N=N_{c}+N_{g}$ gravitationally interacting particles is written as

$$
\begin{gathered}
\frac{d \mathbf{v}_{i}}{d t}=\sum_{j=1, j \neq i}^{N} \mathbf{f}_{i j}, \quad i=1, \ldots, N_{c}, \\
\frac{d \mathbf{v}_{i}}{d t}=-\frac{\nabla p_{i}}{\varrho_{i}}+\sum_{j=1, j \neq i}^{N} \mathbf{f}_{i j}, \quad i=1, \ldots, N_{g}, \\
\frac{d \varepsilon_{i}}{d t}=-\frac{p_{i}}{\varrho_{i}} \nabla \cdot \mathbf{v}_{i}, \quad i=1, \ldots, N_{g}, \\
\varepsilon_{i}=\frac{p_{i}}{(\gamma-1) \varrho_{i}}, \quad i=1, \ldots, N_{g},
\end{gathered}
$$

where $\gamma$ is the adiabatic index, $\varrho_{i}, p_{i}, \varepsilon_{i}, \mathbf{v}_{i}$ are the mass density, gas pressure, specific internal energy, and velocity vector of the $i$-th particle, respectively. The gravitational 
interaction force between $i$-th and $j$-th particles is

$$
\mathbf{f}_{i j}=-G \frac{m_{j}\left(\mathbf{r}_{i}-\mathbf{r}_{j}\right)}{\left|\mathbf{r}_{i}-\mathbf{r}_{j}+\delta\right|^{3}},
$$

where $G$ is the gravitational constant, $m_{j}$ is the mass of $j$-th particle, $\delta$ is the gravitational softening length at short distances, the radius-vector $\mathbf{r}_{i}(t)=\int \mathbf{v}_{i} d t$ determines the position of the $i$-th particle in space.

The behavior of the collisionless components of the colliding galaxies is described by the vector equation of motion (1) governed by common gravity of interaction systems.

The dynamics of hot halo gas is described by the set of hydrodynamical equations (2) - (4) which includes a vector equation of motion of gas particles and energy conservation equation. Only two physical source terms are included into the equation of motion of gaseous particles: an acceleration due to a common gravity from gaseous, as well as collisionless components (5), and acceleration arising from local gradients of gas pressure.

In simulations, conducted here, we adopt adiabatic index $\gamma=1,2$. The choice of the parameter $\gamma$ strongly influences the thermodynamic properties of the gas. If the parameter $\gamma$ is close to 1 (see the equation of state in $(2)$ ), we reduce the efficiency of gas heating due to nonlinear waves, since we have an isothermal process. Such an approach is used as the simplest cooling model in various studies on the dynamics of astrophysical gas [10-13]. A more realistic consideration of thermal processes requires a cooling function that depends on temperature and density [14-17] and we plan to investigate this issue in the future. A correct description of the gas cooling is possible for multicomponent models that take chemical reactions into account, as is done in modelling molecular clouds in S-galaxy discs [18-20]. A precise taking into account of gas cooling should include the multi-component models and radiative transfer (see, e.g. [15]), and we leave this for future consideration.

Results of fluid dynamical and N-body simulations sensitively depend on the initial conditions. To build the initial conditions for our simulation runs, we use the method, described in $[21,22]$. To avoid an impact of unrelaxed initial conditions, we let collisionlessgaseous halos to evolve during 6 Gyr prescribing the the initial velocities and the impact parameter of the colliding systems.

A schematic picture of our numeral simulations is shown in Fig. 1. At the beginning of simulations two colliding galaxies that have masses $M_{1}$ and $M_{2}$ move with the velocities $\mathbf{V}_{1}$ and $\mathbf{V}_{2}$, which in general case have different values and different directions. In the inertial reference frame that moves with the velocity equal to $\mathbf{V}^{*}=\left(\mathbf{V}_{1}+\mathbf{V}_{2}\right) / 2$, both galaxies will move in the parallel directions with the velocities $\mathbf{V}=\left(\mathbf{V}_{1}-\mathbf{V}_{2}\right) / 2$ and $-\mathbf{V}$, correspondingly, and will have some impact parameter $s(\mathrm{kpc})$. The halos of both colliding galaxies have the collisionless as well as the gaseous components with masses $\left(M_{1 c}, M_{2 c}\right)$ and $\left(M_{1 g}, M_{2 g}\right)$ correspondingly. We assume in our simulations that the radius of the gaseous halo is less than the radius of the collisionless one, i.e., $R_{g}<R_{c}$. In this paper, we limit our consideration by the collision of two equal mass galaxies.

\section{Numerical Code and Parameters}

To approximate numerically the spatial derivatives in the system of equations (2) - (4), we use the smoothed particle hydrodynamics approach (SPH) [23-25], while calculating the gravitational interaction of gas as well as of the collisionless particles directly, we use so-called Particle-Particle algorithm (5). To perform the collisional dynamics between the 


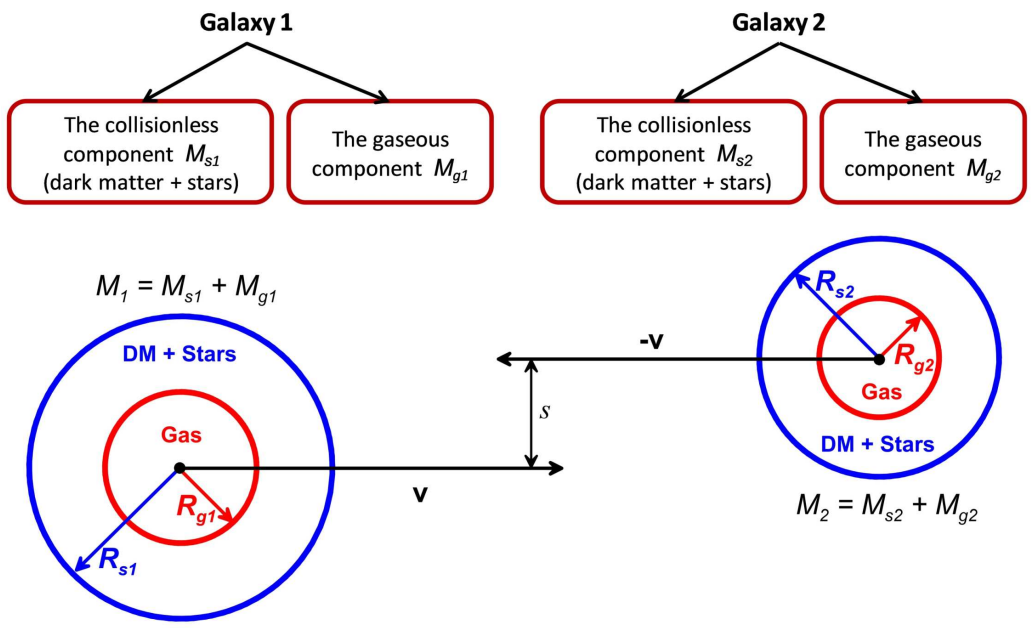

Fig. 1. The computational model of the collisions of live halos of galaxies

halos of galaxies we use KDK (Kick-Drift-Kick) scheme for SPH + N-body code with a parallel realization of the numerical algorithm for the hybrid computational systems CPU + GPU. The calculations were carried out on GPU Nvidia Tesla computers: K20, K40, K80. As it was mentioned above, we perform N-body simulations using direct integration between the gravitating particles avoiding thus possible errors associated with calculation of gravitational forces by the Tree algorithm [26, 27]. The Particle-Particle approach provides the best accuracy for the total gravitational force calculation. We use a parallel algorithm to solving the N-body + SPH problems based on the hybrid technology OpenMP-CUDA with the number of particles $N=10^{5}-10^{7}$. A detailed description of the numerical algorithms SPH and N-body is given in papers [28, 29]. In the papers [30,31] it was also noted that the result of the parallel software implementation and its efficiency depends on the details of the code and on the sequence of the numerical operations. Fig. 2 shows the sequence of running global CUDA kernels for the parallel algorithm to solve the N-body + SPH problems. Our computer algorithm consists of the following global CUDA kernels:

- The Sorting Particles (SP) is a set of CUDA Kernels to determine the particles' numbering and number of particles in three-dimensional grid cells.

- The Density Computation (DC) is a CUDA Kernel for density calculation.

- The Hydrodynamics Force Computation (HFC) is a CUDA Kernel for the hydrodynamic forces calculation.

- The Gravity Force Computation (GFC) is a CUDA Kernel for the gravitational forces calculation between SPH and N-body particles.

- The Update System (US) is a CUDA Kernel to update particle characteristics in accordance with the equations (1) - (3). The kernel has two states \{predictor, corrector\}.

We use different time steps for SPH and N-body algorithms, as shown in the Fig. 2. This approach can significantly reduce the computational time, since the gravitational force calculation (GFC) between the particles occurs only once in 3 - 100 hydrodynamic time steps. We estimate the relative error in calculating the parameters of SPH-particles within $10^{-4}$ for such an algorithm. We use the following units: the gravitational constant $G=1$, the unit of mass is equal to $M=2,2 \times 10^{9} M_{\odot}$, and the unit of length is equal to $L=1 \mathrm{kpc}$, which gives for the unit of time the value of $10 \mathrm{Myr}$, and for the velocity the \& Computer Software (Bulletin SUSU MMCS), 2019, vol. 12, no. 2, pp. 123-135 


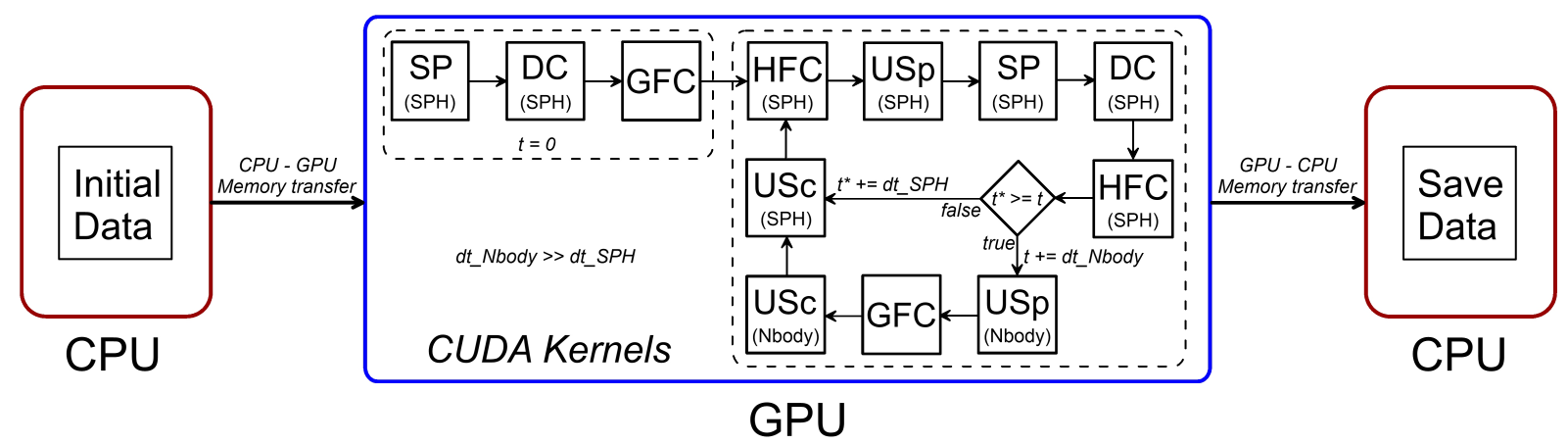

Fig. 2. Flow diagram for the calculation module. The parentheses indicate for which particles (SPH or N-body) CUDA kernels are used

unit of $100 \mathrm{~km} / \mathrm{sec}$. The set of parameters of the colliding galaxies in dimensionless units we used in our numerical simulations is summarized in Table.

Table

Parameters of models in our numerical experiments

\begin{tabular}{|c|c|c|c|c|c|}
\hline $\begin{array}{c}\text { Number } \\
\text { of run }\end{array}$ & $\begin{array}{c}\text { Mass of halo } \\
(\mathrm{DM}+\text { Stars })\end{array}$ & $\begin{array}{c}\text { Mass of halo } \\
(\mathrm{Gas})\end{array}$ & $\begin{array}{c}\text { Initial velocity of } \\
\text { galaxies }\end{array}$ & $\begin{array}{c}\text { Impact } \\
\text { parameter }\end{array}$ & $\begin{array}{c}\text { The result } \\
\text { of the collision }\end{array}$ \\
\hline 1 & 50 & 7,5 & 6 & 0 & scattering \\
\hline 2 & 50 & 7,5 & 6 & 3 & scattering \\
\hline 3 & 50 & 7,5 & 15 & 0 & scattering \\
\hline 4 & 50 & 7,5 & 30 & 0 & scattering \\
\hline 5 & 50 & 7,5 & 30 & 3 & scattering \\
\hline 6 & 50 & 15 & 10 & 0 & scattering \\
\hline 7 & 50 & 15 & 15 & 0 & scattering \\
\hline 8 & 50 & 15 & 30 & 0 & scattering \\
\hline 9 & 10 & 1,5 & 2 & 0 & merging \\
\hline 10 & 10 & 1,5 & 2 & 3 & merging \\
\hline 11 & 10 & 1,5 & 2 & 6 & scattering \\
\hline 12 & 10 & 1,5 & 3 & 0 & scattering \\
\hline 13 & 10 & 1,5 & 3 & 3 & scattering \\
\hline
\end{tabular}

\section{Collisional Model of Tutukov et al [8]}

A collision of two gaseous halos of galaxies was studied by A.V. Tutukov et al $[7,8]$. These authors considered several scenarios of collisions of gaseous halos of galaxies in models with the fixed gravitational potential. Tutukov et al found that for certain velocities of the collision, there is possible a formation of third galaxy made of gas.

Besides the assumption of fixed gravitational potentials of galaxies, A.V. Tutukov et al assumed also that fixed gravitational potentials move towards each other with a constant velocities during the collision of the galaxies. Briefly, the basic assumptions of [7] can be summarized as follows:

1. The collisionless components of halos are assumed to be homogeneously distributed within a sphere of some radius $R_{s}$ and remain intact during a collision. 
2. The velocities of the collisionless components remain constant in a process of a collision. 3. Masses of fixed collisionless halos, and the gaseous ones are equal $M_{g}=M_{s}$.

There is interesting to study the possibility of the formation of third galaxy from gas that is lost in a process of collision of galaxies, predicted in the model of Tutukov et al in a more realistic self-consistent model when each galaxy is modeled by a "live" collisionless and gaseous components joined by a common gravity as it shown in (Fig. 1).

\section{Results of Our Modelling}

Fig. 3 (frames (a) and (e) at $t=0$ ) shows the initial density distributions in both galaxies for our "fiducial" case, the collision of two equal mass galaxies containing amount of hot gas equal to fifteen percent of mass of the collisionless halos (number of run 1 in the Table). At the beginning, gas as well as the collisionless particles of both halos are symmetrically distributed around their centers of mass. The galaxies start to run towards each other with zero impact parameter and with the initial velocity equal to $600 \mathrm{~km} / \mathrm{sec}$. At the initial stages of collision (time $t=19$, snapshots (a) and (e) of Fig. 3), a common envelope containing both stars and gas is formed, and the powerful shock fronts appear in the gaseous components of the halos. The lifetime of the shock fronts though does not exceed 10 Myr. Later, after time $t=26$, in the new cone-like shock fronts arise caused by supersonic flows of the gaseous components moving through gravitational potential wells of the galaxies. The estimated lifetime of these secondary conical fronts is about $50 \mathrm{Myr}$.

The conical fronts, although less prominent, are seen in the collisionless components as well. Qualitatively, can be explained by the impact on each particle caused by the gravitational field of another galaxy in the direction of the line of the collision of two galaxies.

The cones in the gaseous components are more prominent, and are formed from hot gas that passed through the shock fronts. At later stages of the collision (see Fig. $3(\mathrm{~h})$ ), a gaseous bridge connecting two moving away galaxies is formed.

By our estimate, about 40 percent of stars and about 70 percent of gas is expelled into the intergalactic space during the head-on collision.

Let's discuss in more detail the structure of the shock wave in the region of the formation of the cone-like shock front as shown in Fig. 3 (f). Fig. 5 illustrates the profiles along the axis of collision for density, $\varrho$, the speed of sound, $c_{s}$, and gas velocity $u_{*}$, measured relative to the shock front. The front itself is located around $x=11,35$, where $c_{s}=u_{*}$, and where the density jump is observed. Fig. 4 shows the structure of flow in the vicinity of the cone-like shock wave.

Fig. 6 shows the results of our simulations in case of the non-central collision of two equal mass galaxies with the impact parameter equal to $3 \mathrm{kpc}$. In this case, the deceleration of two colliding galaxies is smaller, and each galaxy gets its own angular momentum due to a non-central galaxy-galaxy interaction. As one can see from Fig. 6, the ejected gas and the collisionless particles are also located in two cones, and estimated masses of gas and the collisionless particles of gas and stars expelled into the intergalactic space are about 40 and 20 percent correspondingly.

Our simulations thus show that a considerable amount of hot halo gas becomes gravitationally unbound after the galaxy-galaxy collisions. We confirm therefore the envision of Norman and Silk [1] that at early stages of evolution collisions of hot gaseous 

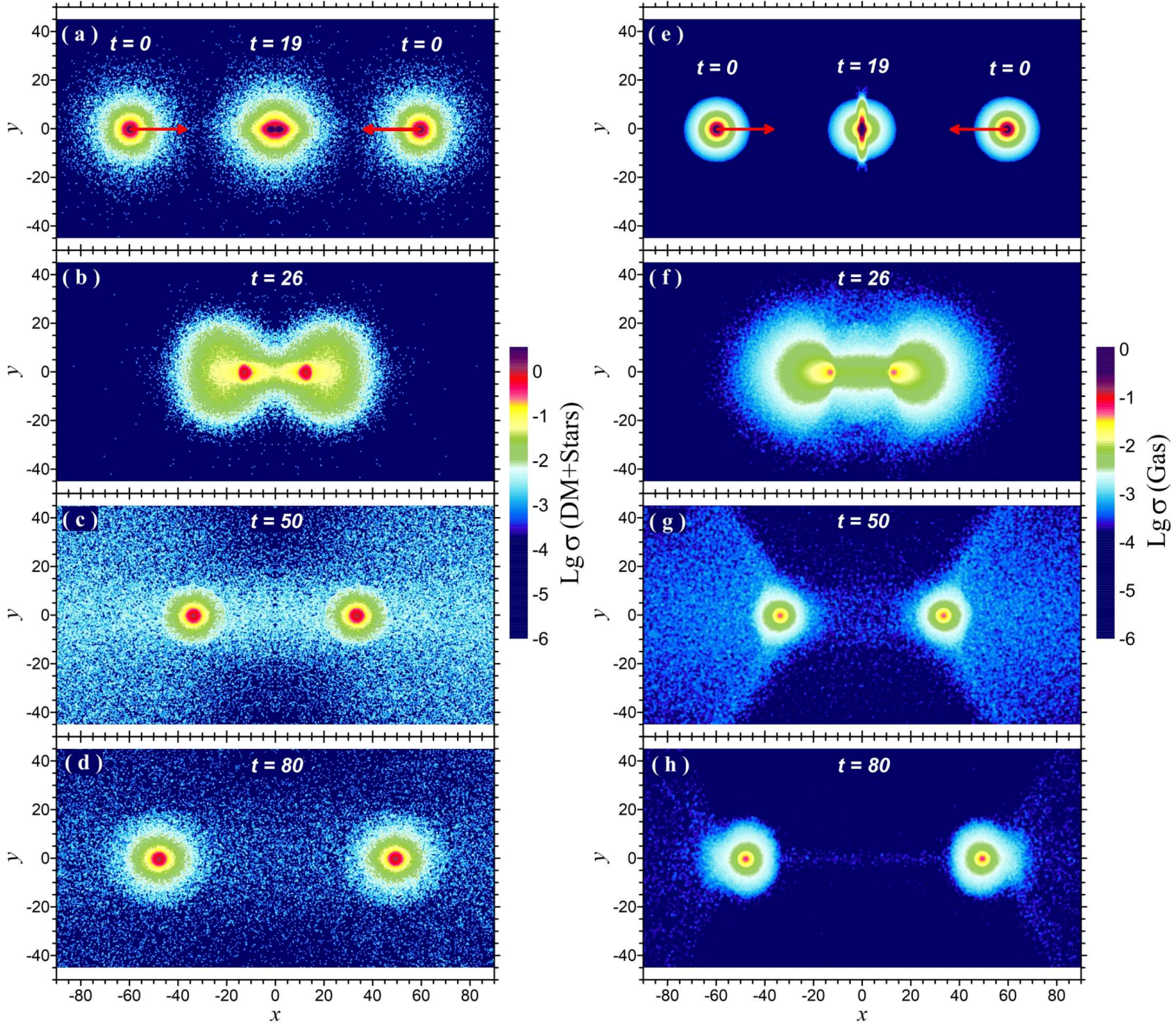

Fig. 3. Surface density distributions of galaxies at different times of their head-on collision. Left panels, collisionless component, right panels, gas surface density distributions

halos of galaxies was a powerful mechanism of expelling of large amounts of gas into the intergalactic space, and also a mechanism of exchange of gaseous components between the colliding galaxies.

The SPH-method allows to follow the dynamics of particles belonging previously to each galaxy. We find that the colliding galaxies exchange $80 \%$ of their gaseous components and about $5 \%$ of their collisionless components during a head-on collision. In case of a noncentral collision, the percentage of exchanged gaseous component is about $30 \%$ and about $1 \%$ of their collisionless component. This conclusion, in particular, makes improper a usage of the "closed box" models in studying the chemical evolution of galaxies.

Goulding et al [32] recently studied the properties of hot gas in a sample of local elliptical galaxies. They conclude that within the inner $30 \mathrm{kpc}$ region, the early type galaxies contain pressure supported hot gas, and the majority of galaxies demonstrate evidence for an additional gas heating. As one can see from our numerical simulations, collisions of galaxies can be a natural mechanism for such an extra-heating, and provides additional argument in favor of collisions of galaxies in the past. Remarkably, Chandra 


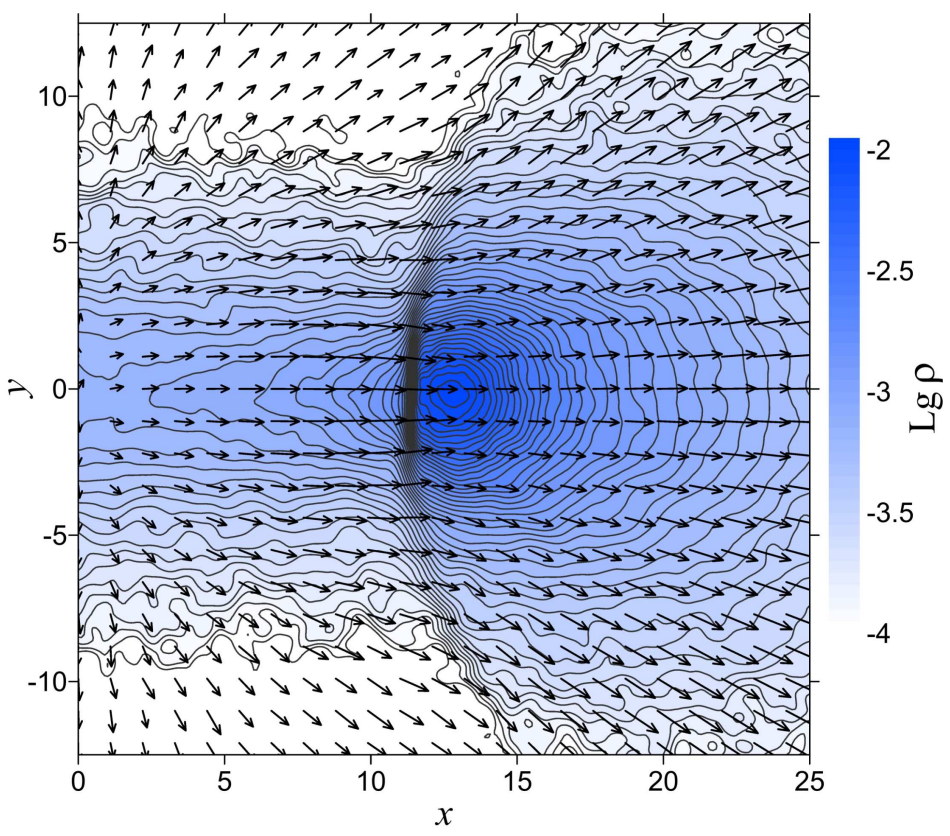

Fig. 4. Structure of the shock front along the axis of the collision taken at $t=26$. The density is normalized to $2,2 M_{\odot} / \mathrm{pc}^{3}$

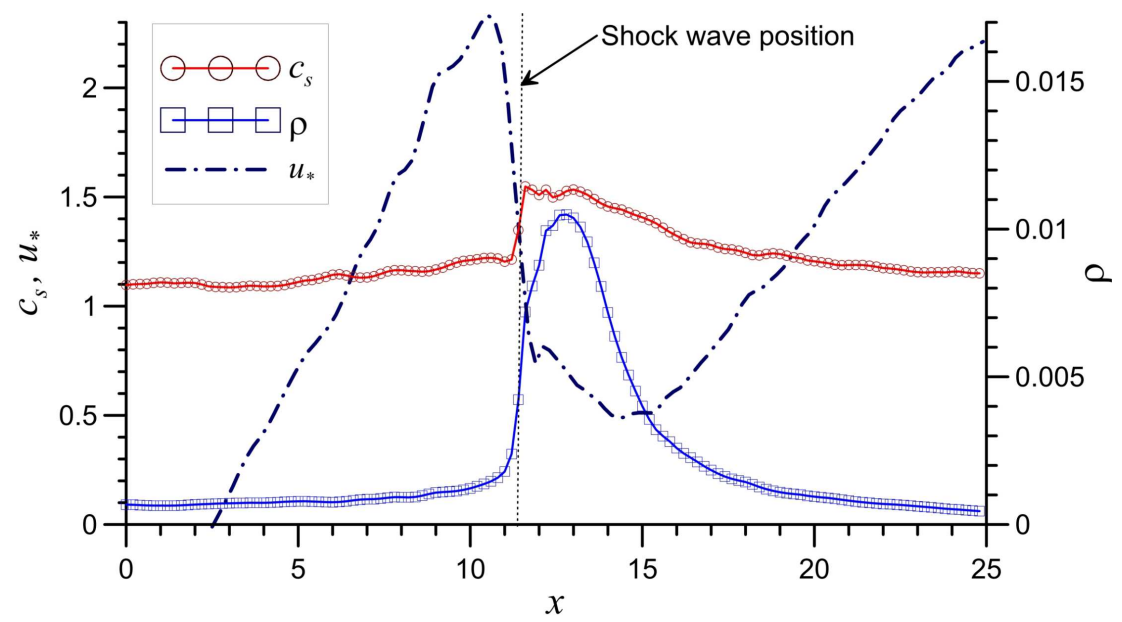

Fig. 5. Distributions of density and the velocity field of gas taken at time equal to $t=26$ within the cone-like shock front

ACIS-S images of local ellipticals demonstrate that hot gas in many systems is located in cones of the type predicted in our simulations. Examples are the galaxies NGC 0383, NGC 1132, and NGC 7618. Fig. 2 in the article [32, p. 10] shows an exposure-corrected ACIS-S Chandra X-ray image of NGC 7618. A cone-like distribution of hot gas, similar to that observed in our numerical simulations, is seen in this image, (see Fig. 7).

\section{Conclusions}

We report about the development of the software package, allowing to perform massive parallel N-body + SPH simulations using OpenMP-CUDA tools of our realizations of computational fluid dynamics $[28,29]$. The software package allows to perform massive 

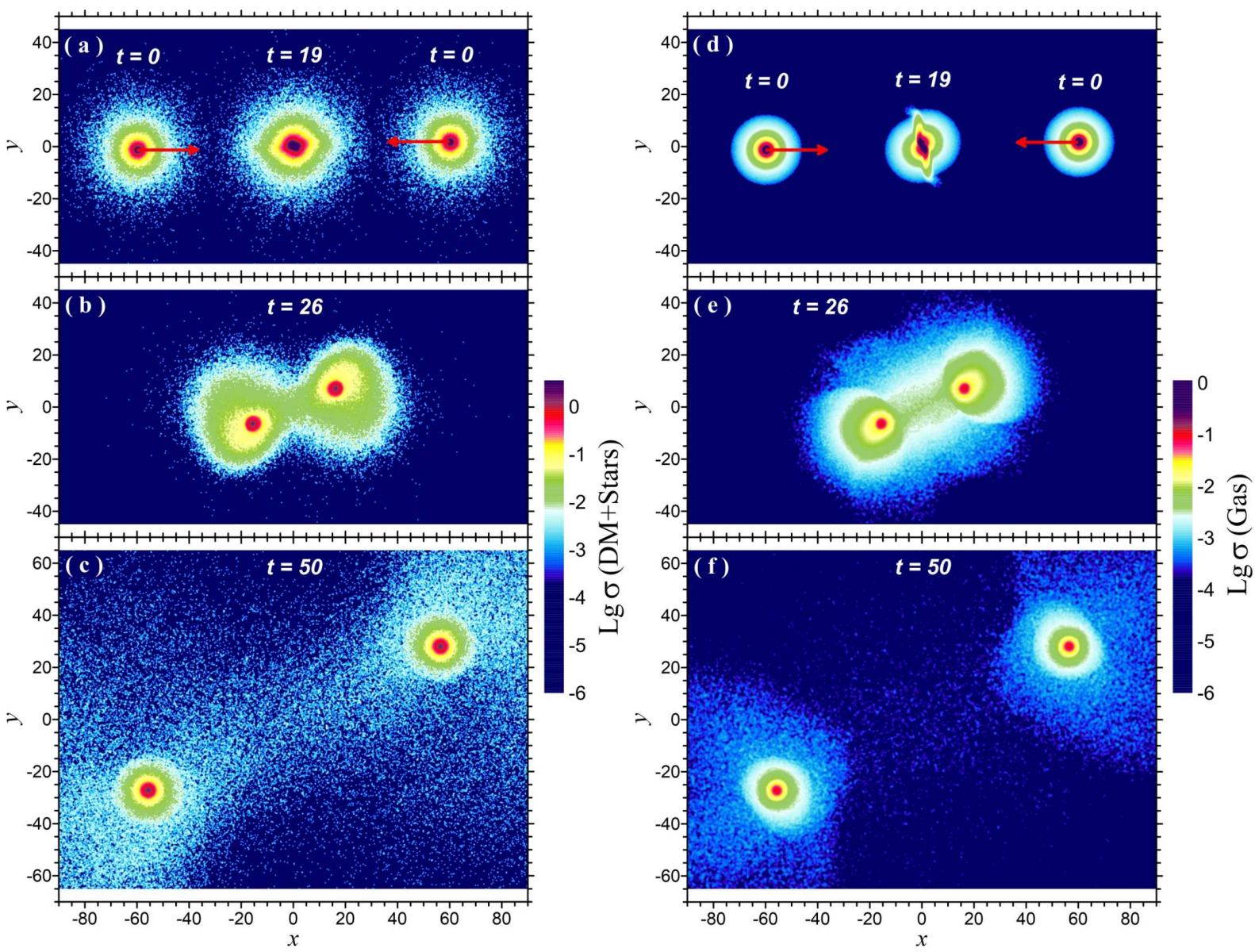

Fig. 6. The surface density distributions for non-central collision of galaxies (impact parameter $s=3$ ) taken at different moments of time. In the left panel the collisionless component is plotted. The gaseous component is plotted in the right panel

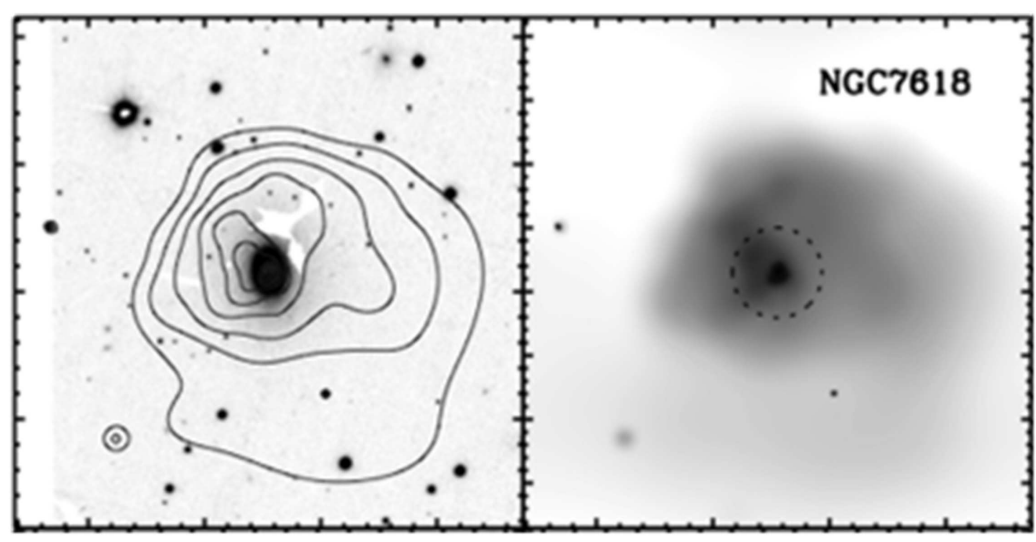

Fig. 7. The logarithm of the adaptively smoothed and exposure corrected Chandra ACIS-S X-ray image of the galaxy NGC 7618 (see e.g. [32]). A cone of hot X-ray gas is clearly seen in the image 
parallel simulations of realistic dynamics of gaseous-collisionless systems joined by their common gravity. As the example of application of our software package, we perform massive parallel three-dimensional N-body and gas-dynamical simulations to study the dynamics of hot halo gas in the colliding galaxies. We model two different collisional scenarios - the "bull-eye" and the non-central collision to check whether the collisions of galaxies in their past can explain observed deficit of baryons in the Universe. The results of our modelling can be summarized as follows:

1. Contrary to the conclusion made by [7] and [8], we demonstrate that the self-consistent consideration of the problem and taking into account the self-gravity of the gaseous and the collisionless components of the galactic halos does not lead to the formation of a gaseous "protogalaxy" after the collision.

2. We demonstrate that up to 70 percent of the hot halo gas can be expelled into the intergalactic region, and confirm the prediction of [1] that a substantial amount of hot gas can be released into intracluster space during galactic collisions which can explain the observed deficit of baryons in today's Universe.

3. We show that a considerable amount of hot halo gas exchanges between the colliding galaxies, thus making galactic collisions the important mechanism of mass and heavyelements exchange in the Universe.

4. We show that the cone-like shock fronts are formed in hot halo gas during the collisions, which can explain the existence of hot gaseous cones observed in the X-ray emission of some nearby elliptical galaxies.

Acknowledgements. AVK and SSK acknowledges the Ministry of Science and Higher Education of the Russian Federation (government task, project No. 2.852.2017/4.6) for the financial support of the development of the software and numerical simulations. VIK thanks Russian Science Foundation (grant No. 18-12-00213) for the financial support of the development of models of galaxies and the analysis of the results of numerical simulations. Authors thank T. Girard for valuable comments. The research is carried out using the equipment of the shared research facilities of HPC computing resources at Lomonosov Moscow State University supported by project RFMEFI62117X0011.

\section{References}

1. Norman C., Silk J. Interstellar Bullets - H2O Masers and Herbig-Haro Objects. Astrophysical Journal, 1979, vol. 228, pp. 197-205. DOI: 10.1086/156836

2. Mathews W.G., Brighenti F. Hot Gas in and Around Elliptical Galaxies. Annual Review of Astronomy and Astrophysic, 2003, vol. 41, pp. 191-239. DOI: 10.1146/annurev.astro.41.090401.094542

3. O'Sullivan E., Forbes D.A., Ponman T.J. A Catalogue and Analysis of X-Ray Luminosities of Early-Type Galaxies. Monthly Notices of the Royal Astronomical Society, 2001, vol. 328, pp. 461-484. DOI: 10.1046/j.1365-8711.2001.04890.x

4. Fukugita M., Hogan C.J., Peebles P.J.E. The Cosmic Baryon Budget. The Astrophysical Journal, 1998, vol. 503, pp. 518-530. DOI: 10.1086/306025

5. Fukugita M., Peebles P.J.E. The Cosmic Energy Inventory. The Astrophysical Journal, 2004, vol. 616, pp. 643-668. DOI: 10.1086/425155

6. Sinha M., Holley-Bockelmann K. Numerical Simulations of Hot Halo Gas in Galaxy Mergers. Galaxy Evolution: Emerging Insights and Future Challenges ASP Conference Series, 2009, vol. 419, pp. 263-266. DOI: $10.1063 / 1.3458479$ 
7. Tutukov A.V., Lazareva G.G., Kulikov I.M. Gas Dynamics of a Central Collision of Two Galaxies: Merger, Disruption, Passage and the Formation of a New Galaxy. Astronomy Reports, 2011, vol. 55, pp. 770-783. DOI: 10.1134/S1063772911090083

8. Vshivkov V.A., Lazareva G.G., Snytnikov A.V., Kulikov I.M., Tutukov A.V. Hydrodynamical Code for Numerical Simulation of the Gas Components of Colliding Galaxies. The Astrophysical Journal Supplement, 2011, vol. 194, p. 47. DOI: 10.1088/0067-0049/194/2/47

9. Hwang J.-S., Park C. Effects of Hot Halo Gas on Star Formation and Mass Transfer During Distant Galaxy-Galaxy Encounters. The Astrophysical Journal, 2015, vol. 805, p. 131. DOI: $10.1088 / 0004-637 \mathrm{X} / 805 / 2 / 131$

10. Sawada K., Matsuda T., Hachisu I. Spiral Shocks on a Roche Lobe Overflow in a SemiDetached Binary System. Monthly Notices of the Royal Astronomical Society, 1986, vol. 219, pp. $75-88$. DOI: $10.1093 / \mathrm{mnras} / 219.1 .75$

11. Bisikalo D.V., Boyarchuk A.A., Kuznetsov O. A., Chechetkin V.M. The Effect of Viscosity on the Flow Morphology in Semidetached Binary Systems. Results of 3D Simulations. II. Astronomy Reports, 2000, vol. 44, pp. 26-35. DOI: 10.1134/1.163824

12. Khoperskov S.A., Khoperskov A.V., Eremin M.A., Butenko M.A. Polygonal Structures in a Gaseous Disk: Numerical Simulations. Astronomy Letters, 2011, vol. 37, pp. 563-575. DOI: $10.1134 /$ S032001081108002X

13. Dobbs C.L., Bonnell I.A., Clark P.C. Centrally Condensed Turbulent Cores: Massive Stars or Fragmentation? Monthly Notices of the Royal Astronomical Society, 2005, vol. 360, pp. 2-8. DOI: $10.1111 / \mathrm{j} .1365-2966.2005 .08941 . x$

14. Vasiliev E.O. Thermal Instability in a Collisionally Cooled Gas. Monthly Notices of the Royal Astronomical Society, 2012, vol. 419, pp. 3641-3648. DOI: 10.1111/j.1365-2966.2011.20017.x

15. Khoperskov S.A., Vasiliev E.O., Sobolev A.M., Khoperskov A.V. The Simulation of Molecular Clouds Formation in the Milky Way. Monthly Notices of the Royal Astronomical Society, 2013, vol. 428, pp. 2311-2320. DOI: 10.1093/mnras/sts195

16. Vorobyov E.I., Recchi S., Hensler G. Stellar Hydrodynamical Modelling of Dwarf Galaxies: Simulation Methodology, Tests and First Results. Astronomy and Astrophysics, 2015, vol. 579, pp. 1-23. DOI: 10.1051/0004-6361/201425587

17. Vasiliev E.O., Shchekinov Yu.A. Supernova Remnants in the $\mathrm{H} \alpha$ and $\mathrm{H} \beta$ Lines. Astrophysics, 2017, vol. 60, pp. 1-18. DOI: 10.1007/s10511-017-9458-9

18. Dobbs C.L., Pringle J.E. The Exciting Lives of Giant Molecular Clouds. Monthly Notices of the Royal Astronomical Society, 2013, vol. 432, pp. 653-667. DOI: 10.1093/mnras/stt508

19. Khoperskov S.A., Vasiliev E.O., Ladeyschikov D.A., Sobolev A.M., Khoperskov A.V. Giant Molecular Cloud Scaling Relations: the Role of the Cloud Definition. Monthly Notices of the Royal Astronomical Society, 2016, vol. 455, pp. 1782-1795. DOI: 10.1093/mnras/stv2366

20. Duarte-Cabral A., Dobbs C.L. What Can Simulated Molecular Clouds Tell Us About Real Molecular Clouds? Monthly Notices of the Royal Astronomical Society, 2016, vol. 458, pp. 3667-3683. DOI: $10.1093 / \mathrm{mnras} / \mathrm{stw} 469$

21. Khoperskov A., Bizyaev D., Tiurina N., Butenko M. Numerical Modelling of the Vertical Structure and Dark Halo Parameters in Disc Galaxies. Astronomische Nachrichten, 2010, vol. 331, pp. 731-745. DOI: 10.1002/asna.200911402

22. Khoperskov S., Vasiliev E., Khoperskov A., Lubimov V. Numerical Code for MultiComponent Galaxies: from N-Body to Chemistry and Magnetic Fields. Journal of Physics, 2014, vol. 510, article ID: 012011, 13 p. DOI: 10.1088/1742-6596/510/1/012011 
23. Monaghan J.J. Smoothed Particle Hydrodynamics. Annual Review of Astronomy and Astrophysics, 1992, vol. 30, pp. 543-574. DOI: 10.1146/annurev.astro.30.1.543

24. Monaghan J.J. Smoothed Particle Hydrodynamics. Reports on Progress in Physics, 2005, vol. 68, pp. 1703-1759. DOI: 10.1088/0034-4885/68/8/R01

25. Mokos A., Rogers B.D., Stansby P.K., Dominguez J.M. Multi-Phase SPH Modelling of Violent Hydrodynamics on GPUs. Computer Physics Communications, 2015, vol. 196, pp. 304-316. DOI: $10.1016 /$ j.cpc.2015.06.020

26. Barnes J.E., Hut P. Error Analysis of a Tree Code. Astrophysical Journal Supplement Series, 1989, vol. 70, pp. 389-417. DOI: 10.1086/191343

27. Lukat G., Banerjee R. A GPU Accelerated Barnes-Hut Tree Code for FLASH4. New Astronomy, 2016, vol. 45, pp. 14-28. DOI: 10.1016/j.newast.2015.10.007

28. Khrapov S.S., Khoperskov A.V. Smoothed-Particle Hydrodynamics Models: Implementation Features on GPUs. Communications in Computer and Information Science, 2017, vol. 793, pp. 266-277. DOI: 10.1007/978-3-319-71255-0_21

29. Khrapov S.S., Khoperskov S.A., Khoperskov A.V. New Features of Parallel Implementation of N-Body Problems on GPU. Bulletin of the South Ural State University. Series: Mathematical Modelling, Programming and Computer Software, 2018, vol. 11, pp. 124-136. DOI: $10.14529 / \mathrm{mmp} 180111$

30. Huang S.-Y., Spurzem R., Berczik P. Performance Analysis of Parallel Gravitational N-Body Codes on Large GPU Clusters. Research in Astronomy and Astrophysics, 2016, vol. 16, no. 1, article ID: 11, 11 p. DOI: 10.1088/1674-4527/16/1/011

31. Steinberg O.B. Circular Shift of Loop Body-Programme Transformation, Promoting Parallelism. Bulletin of the South Ural State University. Series: Mathematical Modelling, Programming and Computer Software, 2017, vol. 10, no. 3, pp. 120-132. DOI: $10.14529 / \mathrm{mmp} 170310$

32. Goulding A.D., Greene J.E., Chung-Pei Ma. The MASSIVE Survey. IV. The X-Ray Halos of the Most Massive Early-Type Galaxies in the Nearby Universe. The Astrophysical Journal, 2016, vol. 826, p. 167. DOI: $10.3847 / 0004-637 \mathrm{X} / 826 / 2 / 167$

Received February 14, 2019

УДК 519.6+524.7

DOI: $10.14529 / \mathrm{mmp} 190210$

\section{ЧИСЛЕННОЕ МОДЕЛИРОВАНИЕ ДИНАМИКИ ГАЛАКТИЧЕСКИХ ГАЛО В СТАЛКИВАЮЩИХСЯ ГАЛАКТИКАХ}

\section{C.С. Храпов ${ }^{1}$, А.В. Хоперсков ${ }^{1}$, В.И. Корчагин ${ }^{2}$}

${ }^{1}$ Волгоградский государственный университет, г. Волгоград, Российская Федерация

${ }^{2}$ Южный федеральный университет, г. Ростов-на-Дону, Российская Федерация

Изучено поведение горячего коронального газа в сталкивающихся галактиках с «живыми гало» темной материи на основе параллельного трехмерного моделирования 
самосогласованной динамики N-тел и газа. Рассмотрены различные сценарии галактических столкновений, в том числе центрального и нецентрального, и использовались разные значения начальных скоростей сталкивающихся галактик. Показано, что с учетом собственной гравитации столкновения газообразных и звездных компонентов не приводит к образованию газовой «протогалактики», наблюдаемой в некоторых численных моделях. Также показано, что около шестидесяти процентов горячего газа гало вытесняется в межгалактическое пространство во время столкновения. При численном моделировании наблюдается значительный обмен газом (до 70\% для лобовых столкновений) между двумя сталкивающимися галактиками.

Kлючевые слова: Multi-GPU; OpenMP-CUDA; GPU-Direct; NVIDIA TESLA; задача $N$-тел; SPH; сталкивающиеся галактики.

Сергей Сергеевич Храпов, кандидат физико-математических наук, доцент, доцент кафедры «Информационные системы и компьютерное моделирование», Волгоградский государственный университет (г. Волгоград, Российская Федерация), khrapov@volsu.ru.

Александр Валентинович Хоперсков, доктор физико-математических наук, профессор, заведующий кафедрой «Информационные системы и компьютерное моделирование», Волгоградский государственный университет (г. Волгоград, Российская Федерация), khoperskov@volsu.ru.

Владимир Иванович Корчагин, доктор физико-математических наук, ведущий научный сотрудник, Южный федеральный университет (г. Ростов-на-Дону, Российская Федерация), vkorchagin@sfedu.ru.

Поступила в редакиию 14 февраля 2019 г. 